\title{
Metal-Insulator Transition Temperatures and Excitonic Phases in Vanadium Oxides
}

\author{
A. L. Pergament \\ Physics and Technology Department, Petrozavodsk State University, Petrozavodsk 185910, Russia \\ Correspondence should be addressed to A. L. Pergament, aperg@psu.karelia.ru
}

Received 8 August 2011; Accepted 4 September 2011

Academic Editors: S. Balamurugan and S. Nagata

Copyright () 2011 A. L. Pergament. This is an open access article distributed under the Creative Commons Attribution License, which permits unrestricted use, distribution, and reproduction in any medium, provided the original work is properly cited.

The possibility of applying the excitonic insulator model to the description of metal-insulator transitions in vanadium oxide Magneli phases is investigated. Based on the Animalu transition metal model potential, the equation for the constant of Coulomb interaction in the theory of excitonic insulator is modified. It is shown that this theory allows the transition temperatures of all the oxides to be calculated. The conformity of the theory with the experimental data concerning the effective mass values for electrons in vanadium oxides is discussed.

\section{Introduction}

Studies of metal-insulator transitions (MITs) challenged the well-established division of materials into metals and dielectrics according to the type of electron spectrum and occupation of a band by collective electrons [1]. At present, one can find quite a few examples of materials "violating" the abovementioned division. Among those are compounds exhibiting MITs and revealing properties of either a metal in certain external conditions (temperature, pressure) or an insulator in other conditions. The transition between these two states is generally followed by a drastic change of electrical conduction (by a few orders of magnitude) as well as of other physical characteristics.

A lot of transition- and rare-earth-metal compounds undergo insulator-metal transitions at a particular temperature, $T_{t}$. Of vanadium oxides within the narrow composition range, from $\mathrm{V}_{2} \mathrm{O}_{3}$ to $\mathrm{V}_{6} \mathrm{O}_{13}$ (the "Magneli phases" $\mathrm{V}_{n} \mathrm{O}_{2 n-1}$, $n=2-9$ ), at least eight show such transitions at different temperatures [1]. Vanadium dioxide could be formally included in this pattern with $n=\infty$, whereas $\mathrm{V}_{6} \mathrm{O}_{13}$ belongs to another homology series (the so-called "Wadsley phases" $\mathrm{V}_{2 n} \mathrm{O}_{5 n-2}$ ), the limiting term of which with $n=\infty$ is the insulator $\mathrm{V}_{2} \mathrm{O}_{5}$.

The surprising thing is that the MIT temperatures in vanadium oxides, as well as in other oxides of transition metals, are not calculated in the context of current theories, unlike superconductivity, where the BCS formula predicts the value of $T_{c}$ of many superconducting materials with sufficient accuracy, at least, for elementary metals and some compounds, except for the high- $T_{c}$ superconductors.

In the present work I will try to give some particular formulae allowing the MIT temperature to be calculated for transition metal compounds, vanadium oxides, in the first place. As a matter of fact, there is no end of "true stories" in the world, in no way remarkable among them. I would not have bothered the reader with mine, had it not been for papers [1-4] which played a special part in all of this. My intention here is to provide some general formulae for calculating $T_{t}$ of vanadium oxides on the basis of the excitonic insulator model [2-8], using the model pseudopotential concept [8].

\section{General Theory of an Excitonic Insulator}

To gain an insight into a possible electron spectrum, use could be made of the scheme offered in [1] that enabled one to calculate with a high degree of accuracy the Neel temperature, $T_{N}$, for all vanadium oxides of the Magneli series $[1,2]$. Each vanadium ion can give for a chemical bond 5 electrons, of which two supplement the $p$-shell of each of the $(2 n-1)$ oxygen ions, and, therefore, the number of band electrons per vanadium atom, $\rho$, is easy to obtain as

$$
\rho=\frac{5 n-2(2 n-1)}{n}=\frac{n+2}{n} .
$$


It is relation (1) that we will apply to calculate the free electron density (in a metal phase) for each of the compounds in question.

The concept is based on the excitonic insulator model often used to describe MITs in various systems, including, for instance, doped chalcogenides of Sm [9], potassium molybdenum bronzes [10], and a number of other markedly different systems [11-14], including vanadium oxides [2$4,8,15]$.

In the early studies devoted to the excitonic insulator theory (see, e.g., [5-7] and references therein), it was shown that the energy gap width of an excitonic insulator is given by a simple relation that is actually quite similar to the BCS formula:

$$
\Delta=\hbar \omega_{0} \exp \left(-\frac{1}{\mu}\right)
$$

where the parameter $\omega_{0}$ is proportional to the plasma frequency of free carriers, and $\mu$ is the constant of Coulomb attraction. In the weak-interaction approximation in an ideal model, the latter can be written as $[5,7]$

$$
\mu=\alpha \ln \alpha^{-2}
$$

where $\alpha$ is a nondimensional variable proportional to $\rho^{-1 / 3}$; in fact, $\alpha=\left(\pi a_{\mathrm{H}} k_{\mathrm{F}}\right)^{-1}$, with $a_{\mathrm{H}}$ being the effective Bohr radius, and $k_{\mathrm{F}}$ - the Fermi wave vector. Knowing the specific gravity for each oxide, as well as the value of $\rho$, it is possible to determine the charge carrier density from (1), and $k_{\mathrm{F}}$ is just proportional to the latter to the power $1 / 3$.

The precise equation for the constant of Coulomb interaction is [16]

$$
\mu=\alpha \int_{0}^{2 k_{\mathrm{F}}} \frac{d k}{k \varepsilon(k)}=\alpha \frac{\Omega}{4 \pi} \int_{0}^{2 k_{\mathrm{F}}} U(k) k d k .
$$

Here $\varepsilon$ is the dielectric function of free carriers, and $\Omega$ is a normalizing volume. If we take the potential of Coulomb interaction between two-point charges as $U(k)=$ $(4 \pi / \Omega) /\left(k^{2}+\lambda^{2}\right)$, then it is straightforward to obtain an equation for $\mu(\alpha)$ from expression (4). The result is as follows:

$$
\mu=\alpha \ln \left(\frac{1}{\alpha}+1\right)^{2}
$$

Equation (5) virtually agrees with (3) in the limit of $\alpha \rightarrow 0$, but at high values of $\alpha$ (corresponding to low electron densities), the gap widths obtained from (2) differ significantly in the cases of $\mu$ calculated using (3) and (5), see [8] and curves 1 and 2 in Figure 1.

Now we consider a system in which the normal ground state of each atom is a spherically symmetric one-electron $s$-state. The exact potential of such an atom is just the emptycore pseudopotential:

$$
U_{\mathrm{EC}}(k)=\frac{4 \pi}{k^{2} \varepsilon(k) \Omega} \cos (k R) .
$$

Here $R$ is the radius of the empty core (for ordinary electronhole interaction, it is equal to the Bohr radius $a_{\mathrm{H}}$ if $\varepsilon$

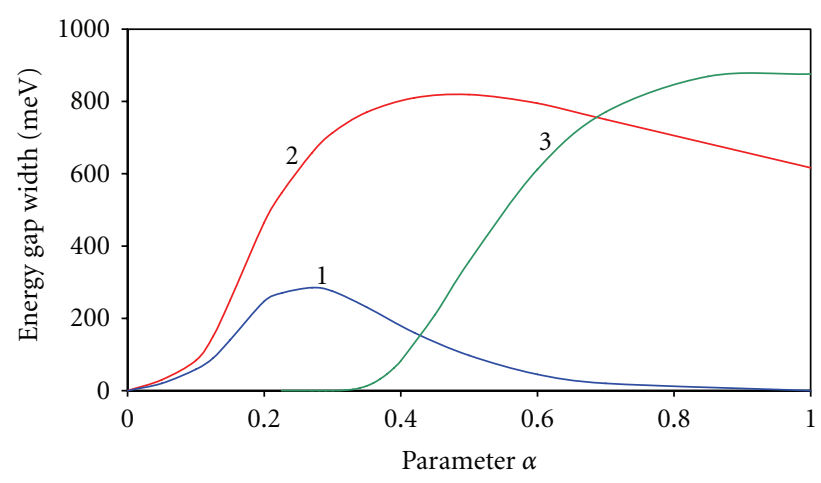

Figure 1: $\Delta$ as a function of $\alpha$. 1: with $\mu$ from (3); 2: (5); 3: (7) with $R=1$ a.u. Curve 3 tends to zero at $\alpha_{c}=0.3-0.4$ which corresponds to the Mott criterion (8).

and $m^{*} / m_{e}$ are equal to unity). The dielectric response $\varepsilon(k)$ characterizes the shielding effect in the Hartree-Fock approximation with the Lindhard factor $g(k)=1-k^{2} /\left(2 k^{2}+\right.$ $\left.k_{\mathrm{F}}^{2}+2 k_{\mathrm{F}} / \pi\right)$. Replacing the potential in (4) by the form taken from (6), we obtain the final form of the constant $\mu$ and, accordingly, the value of the exciton insulator energy gap width from (2). We thus arrive at the following form of the constant of Coulomb interaction (introducing another nondimensional variable $x=k / k_{\mathrm{F}}$ ):

$$
\mu=\frac{\alpha}{R} \int_{0}^{2} \frac{x \cos (R x / \pi \alpha)}{x^{2}+4(\alpha / R) g(x)} d x .
$$

The results of numerical calculations of $\Delta$, with $\mu$ taken from (7), are presented in [8] and shown to comply in this case with the Mott criterion [14]:

$$
a_{\mathrm{H}}\left(\frac{\rho}{\Omega}\right)^{1 / 3} \approx 0.25,
$$

where $a_{\mathrm{H}}$ is the effective Bohr radius, and $\Omega$ is the volume per one vanadium atom, that is, $(\rho / \Omega)$ is in fact the electron density. This is where the main difference lies between the result obtained here and the ones presented in $[5,7]$ (see Figure 1 and discussion in [8]).

\section{Vanadium Oxides (Magneli Phases)}

Next, it has been suggested in [8] that for rather complicated systems, such as vanadium oxides, it is necessary to use in (4) a more realistic model pseudopotential (MPP), $U_{\mathrm{MPP}}(k)$, as compared to a simple $U_{\mathrm{EC}}(k)$. A model potential of a transition metal introduced by Animalu [17], for instance, could act as such a potential, but the effective mass is not determined in this case and should be considered as a parameter. Note that $m^{*}$ appears in expressions for $\alpha, \omega_{0}$, and $U(k)$ in $(2)$ and (4).

As was briefly discussed in [8], calculations of the interaction constant for vanadium dioxide using (4), with $U(k)$ in the form of the Animalu MPP for $V$ [17] (see the appendix), yield the values of $\Delta$ in the range of 0.1 to $\sim 1 \mathrm{eV}$ (depending on the choice of $m^{*}$ in the range of 1 to $10 m_{e}$ ). This appears 
TABLE 1: Parameters of vanadium oxides.

\begin{tabular}{lccc}
\hline Oxide & $T_{t}, \mathrm{~K}$ & $\alpha$ & $m^{*} / m_{e}$ \\
\hline $\mathrm{VO}$ & Metal & - & - \\
$\mathrm{V}_{2} \mathrm{O}_{3}$ & 150 & 0.462 & 1.14 \\
$\mathrm{~V}_{3} \mathrm{O}_{5}$ & 450 & 0.505 & 0.97 \\
$\mathrm{~V}_{4} \mathrm{O}_{7}$ & 240 & 0.526 & 1.19 \\
$\mathrm{~V}_{5} \mathrm{O}_{9}$ & 130 & 0.541 & 1.41 \\
$\mathrm{~V}_{6} \mathrm{O}_{11}$ & 170 & 0.551 & 1.35 \\
$\mathrm{~V}_{7} \mathrm{O}_{13}$ & Metal & 0.560 & 5 (for $T_{t}=0.1 \mathrm{~K}$ ) \\
$\mathrm{V}_{8} \mathrm{O}_{15}$ & 70 & 0.565 & 1.67 \\
$\mathrm{VO}_{2}$ & 340 & 0.616 & 1.25 \\
$\mathrm{~V}_{6} \mathrm{O}_{13}$ & 150 & 0.714 & 1.67 \\
\hline
\end{tabular}

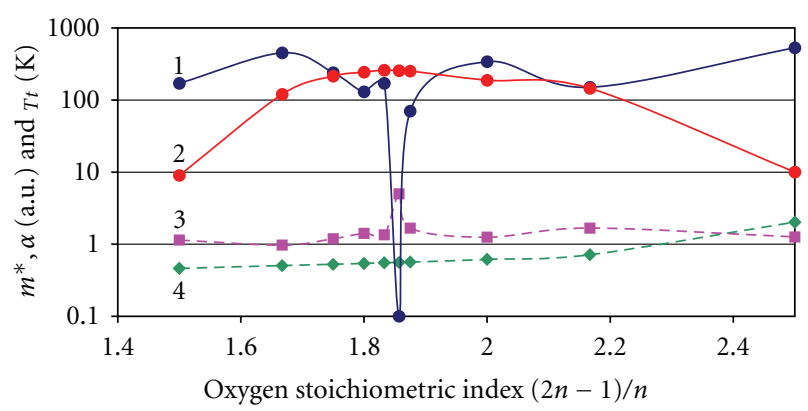

FIGURE 2: 1: experimental transition temperatures for vanadium oxides. 2: calculated $T_{t}$ 's using the Animalu potential with $m^{*}=m_{e}$, but with varied valence $z$ (see the appendix). 3: effective masses (in the units of the mass of a free electron) calculated from the experimental values of $T_{t}$ 's; see also Table 1. 4: parameter $\alpha$ as a function of the oxygen stoichiometric index, calculated using (1) and specific gravities of all the oxides.

to be quite an acceptable agreement if the correlation energy in $\mathrm{VO}_{2}$ is considered to be $\sim 0.1 \mathrm{eV}$ [15]. It can be shown that in this case the temperature of the MIT for $\mathrm{VO}_{2}$ is $T_{t}=$ $\Delta / 3.53 k_{\mathrm{B}}=340 \mathrm{~K}$ (which is equal to the experimental value of $\left.T_{t}[1,14]\right)$ for $m^{*} \sim 1.3 m_{e}$. This estimation of $m^{*}$ appears to be quite reasonable (the experimentally obtained values for vanadium dioxide vary from 0.5 to $\left.3 m_{e}[1-4,18,19]\right)$, and the approach proposed may therefore prove to be an acceptable model for describing a MIT in vanadium and other transition-metal oxides. The same is true for lower oxides $\mathrm{V}_{n} \mathrm{O}_{2 n-1}$ (except for $\left.\mathrm{V}_{7} \mathrm{O}_{13}\right): m^{*} \sim(1-5) m_{e}$, see Figure 2. The experimental values of $T_{t}$ for vanadium oxides are also presented in Table 1, along with the calculated values of $m^{*}$, for the sake of convenience.

Thus, after knowing the effective mass, one can determine the correlation gap and hence the transition temperature as $T_{t}=\Delta / 3.53 k_{\mathrm{B}}$, using (4) and BCS-like (2). It should be however noted that the effective masses are not necessarily known. Even for the much-studied vanadium dioxide, the experimental values obtained by various authors differ nearly by an order of magnitude, as seen above. At the same time the calculated value of $\Delta$ depends rather strongly on $m^{*}$ (see Figure 3). The difficulties and vagueness while determining an effective mass are well known; these are due to both the

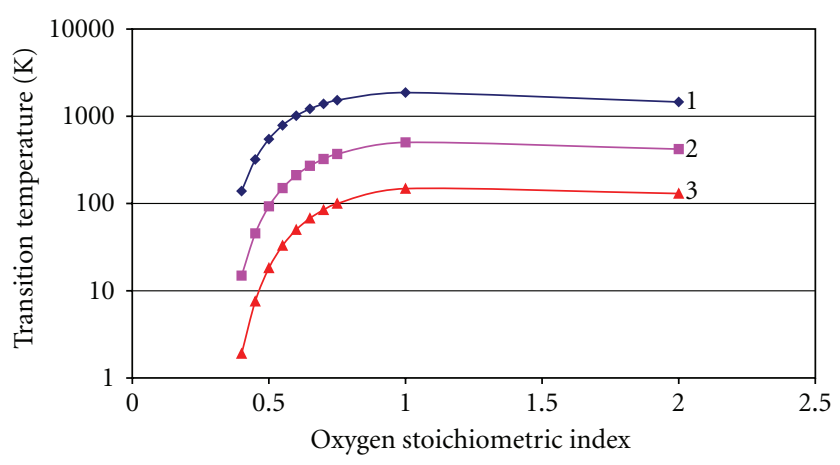

FIgURE 3: Transition temperatures calculated with the Animalu MPP as a function of the oxygen content in vanadium oxides with $m^{*}=1(1), 1.5(2)$, and 2 of $m_{e}$ (3).

quality of a sample and the measuring technique. Optical methods (from measurements of the plasma frequency) and transport oscilloscopic measurements yield as a rule two different magnitudes, which is no wonder, since the values of $m^{*}$ of DOS and band $m^{*}$ should not necessarily coincide [19]. Furthermore, in anisotropic substances this physical quantity depends on direction.

\section{Conclusion}

It has been shown in the present work that using the Animalu's TMMP (transition metal model pseudopotential) for $V$, in the context of the excitonic insulator model, it is possible to calculate correlation energies (from BCS-like formula (2)) and hence the MIT temperature, $T_{t}=\Delta / 3.53 k_{\mathrm{B}}$ for vanadium oxides. Fair agreement between the theory and experiment is observed when choosing the values of the carriers' effective mass in the range from 1 to 5 free electron masses, which does not contradict the well-known experimental data. The sharp increase of $m^{*}$ for $\mathrm{V}_{7} \mathrm{O}_{13}$ may be due to the effective mass divergence close to the MIT (which renders the transition unobservable, drastically decreasing its temperature nearly to absolute zero).

It is more difficult to apply the technique described to multicomponent compounds of transition metals, for example, to CMR manganites or thiospinel $\mathrm{Cu}\left(\mathrm{Ir}_{1-x} \mathrm{Cr}_{x}\right)_{2} \mathrm{~S}_{4}$ [20], where a few kinds of transition metal atoms are available. In general, it is safe to say that the problem discussed is in a certain sense more complicated than the similar problem of Cooper pairing in the theory of superconductivity, where only two electrons and a virtual phonon are involved. In the case of an excitonic phase transition for a d-metal compound, we have to deal with an electron and not with a point-like positively charged hole (or an empty core potential), but we should work with a complex TMMP.

Finally, it should be noted that the problem of the Mott MIT has recently acquired particular significance (or "particular popularity"), mainly because of the intensive studies in the field of MITs in 2D electron gases [21] that are potentially of great importance for the development of nanoelectronics. A purely scientific interest should not be 


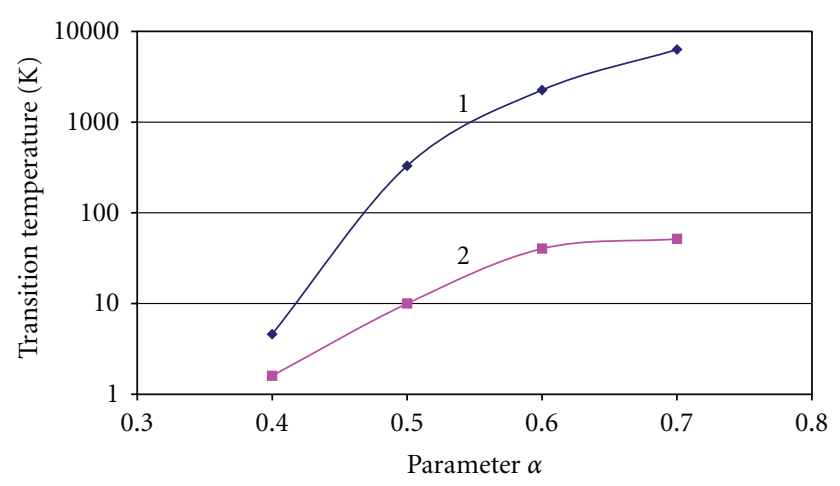

Figure 4: Transition temperature as a function of $\alpha$ : 1 -for $z=5$, 2-for $z=1 ; m^{*}=m_{e}$.

neglected at that. It is important, for instance, to take into account the results obtained here when studying the effect of electric field on the Mott MIT in vanadium oxides [22]. As for the transition of a metal to the excitonic insulator state, it fits well into the framework of a general paradigm for the Mott MITs.

\section{Appendix}

For construction of TMMP, Animalu [17] used the atomic potential in a modified form of the well-known HeineAbarenkov MPP [23]:

$$
V_{\mathrm{MPP}}(r)= \begin{cases}-\sum_{\mathrm{k}} \mathrm{A}_{k} \mathrm{P}_{k}, & \text { for } r \leq R_{m}, \\ -\frac{z}{r}, & \text { for } r>R_{m},\end{cases}
$$

where $P_{k}$ is a component of the angular momentum projection operator, and $A_{k}$ is a parameter representing the depth of a potential well. These parameters, as well as $R_{m}$ and some other constants, were either found $a b$ initio or fitted from the experimental phonon spectra of transition metals [17].

The Fourier-image of this potential, $U_{\mathrm{MPP}}(k)$ represents a rather long expression containing the parameters $A_{k}(k=$ $1,2,3), R_{m}$, and a number of other constants which we will not write here completely. All of these parameters for $V$ were used in the present work, except for $m^{*}$, that is taken in [17] to be equal to unity for all the metals. Also, the valence $z$ (which is obviously taken to be equal to 5 for vanadium metal in [17]) is varied in the present work in compliance with the real value of $z$ of a vanadium ion in a particular $\mathrm{V}_{n} \mathrm{O}_{2 n-1}$ compound (see Figure 2, curve 2, and Figure 4).

The calculating procedure consisted in computing $\Delta$ and then $T_{t}$ for each oxide, using formulae (2) and (4) with $U(k)$ being the Animalu TMMP, varying $m^{*}$ so that the corresponding adjustment yielded finally the real (experimental) value of $T_{t}$. Note that, in principle, one could get some unrealistic magnitudes of an effective mass, for example, 0.001 or 100 of $m_{e}$. Nevertheless, this procedure yielded, as was said, $m^{*} \sim 1 m_{e}$.

\section{Acknowledgments}

This work was supported in part by the Ministry of Education and Science of Russian Federation through the "Scientific and Educational Community of Innovation Russia (2009-2013)" Program, contracts no. 16.740.11.0562, 14.740.11.0895, 02.740.11.5179, 14.740.11.0137, P1156, P1220, and 02.740.11.0395, and the "Development of Scientific Potential of High School" Program, Project no. 12871. The author also is grateful to V. P. Novikova and D. V. Artyukhin for the help with paper preparation.

\section{References}

[1] R. O. Zaĭtsev, E. V. Kuz'min, and S. G. Ovchinnikov, "Fundamental ideas on metal-dielectric transitions in 3d-metal compounds," Soviet Physics Uspekhi, vol. 29, pp. 322-342, 1986.

[2] E. G. Goryachev and S. G. Ovchinnikov, "Electrical and magnetic properties of Magneli phases VnO2n-1," Soviet Physics, Solid State, vol. 20, pp. 2201-2209, 1978.

[3] Y. V. Kopaev and Y. V. Mokerov, "Mechanism of phase transitions in oxides of vanadium and titanium," Doklady Akademii Nauk, vol. 264, pp. 1370-1376, 1982.

[4] B. G. Idlis and Y. V. Kopaev, "On the theory of phase transitions in vanadium oxides $\mathrm{VnO} 2 \mathrm{n}-1$ (magneli phases)," Solid State Communications, vol. 45, no. 3, pp. 301-304, 1983.

[5] L. V. Keldysh and Y. V. Kopaev, "Possible instability of the semimetal state with respect to Coulomb interaction," Soviet Physics, Solid state, vol. 6, pp. 2219-2223, 1964.

[6] D. Jerome, T. M. Rice, and W. Kohn, "Excitonic Insulator," Physical Review, vol. 158, pp. 462-475, 1967.

[7] J. Zittartz, "Theory of the excitonic insulator in the presence of normal impurities," Physical Review, vol. 164, no. 2, pp. 575$582,1967$.

[8] A. Pergament, "Excitonic insulator: dependence of energy gap width on carrier density," Phase Transitions, vol. 83, no. 3, pp. 159-168, 2010.

[9] A. Jung and P. Wachter, "Pressure-driven metal-insulator transition in La- and Tm-doped SmS by exciton condensation," Physica B, vol. 230-232, pp. 725-726, 1997.

[10] W. Fogle and J. H. Perlstein, "Semiconductor-to-metal transition in the blue potassium molybdenum bronze, $\mathrm{K} 0.30 \mathrm{MoO} 3$; example of a possible excitonic insulator," Physical Review B, vol. 6, no. 4, pp. 1402-1412, 1972.

[11] D. Adler, "Mechanisms for metal-nonmental transitions in transition-metal oxides and sulfides," Reviews of Modern Physics, vol. 40, no. 4, pp. 714-736, 1968.

[12] J. Shah, M. Combescot, and A. H. Dayem, "Investigation of exciton-plasma mott transition in Si," Physical Review Letters, vol. 38, no. 25, pp. 1497-1500, 1977.

[13] M. J. Rice and Y. N. Gartstein, "The excitonic ground state of the half-filled Peierls insulator," Journal of Physics Condensed Matter, vol. 17, no. 29, pp. 4615-4620, 2005.

[14] N. F. Mott, Metal_Insulator Transitions, Taylor \& Francis, London, UK, 1974.

[15] A. Pergament, "Metal-insulator transition: the Mott criterion and coherence length," Journal of Physics Condensed Matter, vol. 15, no. 19, pp. 3217-3223, 2003.

[16] V. L. Ginzburg and D.A. Kirzhniz, Eds., High-Temperature Superconductivity, Consultants Bureau, New York, NY, USA, 1982. 
[17] A. O. E. Animalu, "Electronic structure of transition metals. I. Quantum defects and model potential," Physical Review B, vol. 8, no. 8, pp. 3542-3554, 1973.

[18] A. S. Barker Jr., H. W. Verleur, and H. J. Guggenheim, "Infrared optical properties of vanadium dioxide above and below the transition temperature," Physical Review Letters, vol. 17, no. 26, pp. 1286-1289, 1966.

[19] M. M. Qazilbash, M. Brehm, B. G. Chae et al., "Mott transition in VO2 revealed by infrared spectroscopy and nano-imaging," Science, vol. 318, no. 5857, pp. 1750-1753, 2007.

[20] R. Endoh, N. Matsumoto, J. Awaka, S. Ebisu, and S. Nagata, "Metal-insulator transition in the spinel-type $\mathrm{Cu}(\mathrm{Ir} 1-$ xCrx)2S4 system," Journal of Physics and Chemistry of Solids, vol. 63, no. 4, pp. 669-674, 2002.

[21] J. H. Smet, "Metal-insulator transition: a plane mystery," $\mathrm{Na}$ ture Physics, vol. 3, no. 6, pp. 370-372, 2007.

[22] A. L. Pergament, P. P. Boriskov, A. A. Velichko, and N. A. Kuldin, "Switching effect and the metalinsulator transition in electric field," Journal of Physics and Chemistry of Solids, vol. 71, no. 6, pp. 874-879, 2010.

[23] V. Heine, "The Pseudopotential concept," in Solid State Physics: Advances in Research and Applications, H. Ehrenreich, F. Seitz, and D. Turnbull, Eds., Academic Press, New York, NY, USA, 1970. 

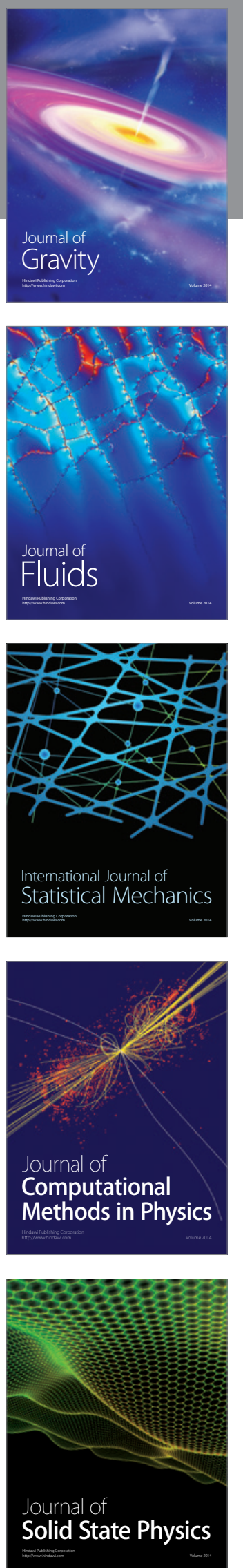
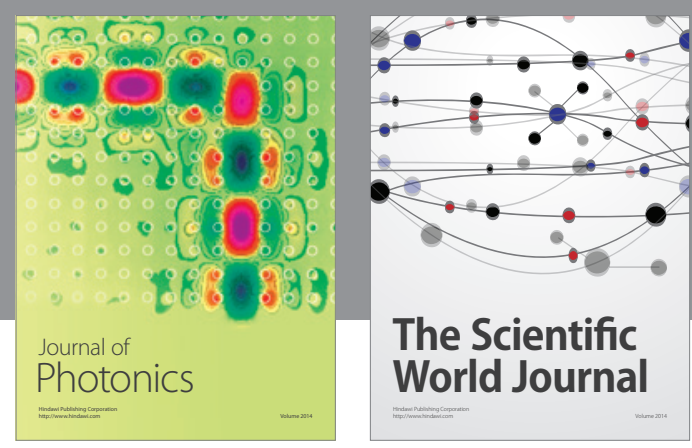

The Scientific World Journal

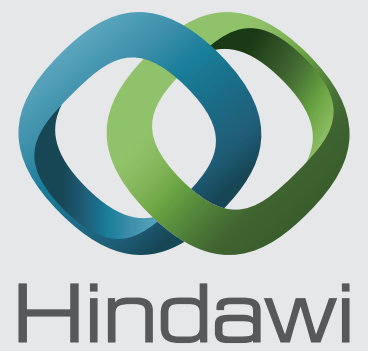

Submit your manuscripts at http://www.hindawi.com
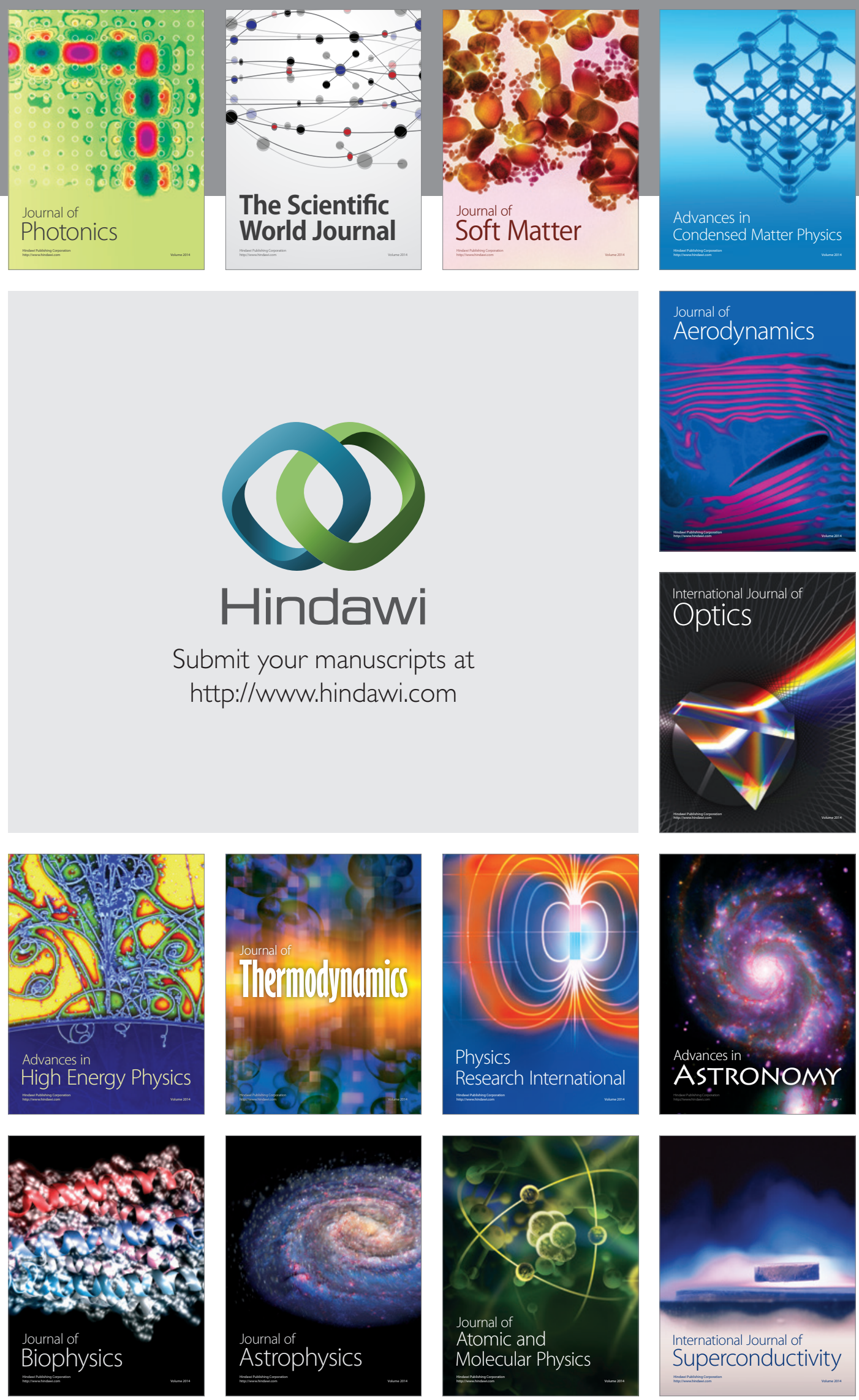
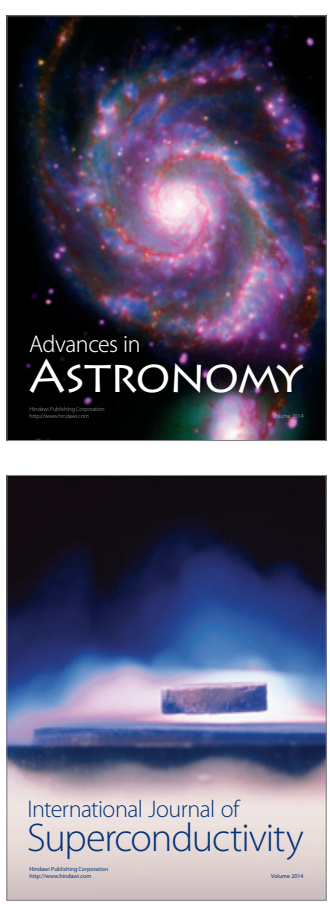